G. DUMITRU
V. ROMANO
H.P. WEBER

G. DUMITRU ${ }^{1, \infty}$

H.P. WEBER ${ }^{2}$

\title{
Model and computer simulation of nanosecond laser material ablation
}

\author{
${ }^{1}$ University of Applied Sciences Aargau, Steinacker Str. 5, 5210 Windisch, Switzerland \\ ${ }^{2}$ University of Bern, Institute of Applied Physics, Sidlerstrasse 5, 3012 Bern, Switzerland
}

\begin{abstract}
Received: 20 October 2003/Accepted: 18 February 2004
Published online: 26 July 2004 • C Springer-Verlag 2004

ABSTRACT A computer model to simulate the evolution of parameters describing laser ablation processes was developed. The absorbed laser energy, the heat diffusion, the phase transformations and the shielding effect of the ablated material were taken into account. The temporal development of the ablated volume, pore depth and extension of the melt zone were calculated for single pulses of 500,100, 20, 5 and $1 \mathrm{~ns}$. Simulations were performed for pulse energies of $50 \mu \mathrm{J}$ and spot diameters of $10 \mu \mathrm{m}$. From temporal evolution curves of the ablated volumes, the stoppage of the ablation process was evidenced before the end of the processing pulse. Comments with respect to optimal pulse duration (in the ns regime) are also formulated.
\end{abstract}

PACS 81.40.Wx

1

\section{Introduction}

Numerous numerical and analytical models have been developed to simulate laser-matter interaction processes: energy absorption, energy transfer to the lattice, phase transformations in the target material, ablation mechanisms and properties of the induced plasma and its interaction with the incident laser beam [1-9]. Model outcomes were also compared with experiments performed on a vast variety of target materials [10-12]. Due to extreme process complexity, most models treat either target-material changes or plasma properties and propagation; some models however address both aspects $[4,6]$.

In this work, efforts are focused on determining the temporal evolution of some parameters that describe the laser ablation process; ns laser pulses and steel as the target material are considered. The global shielding effect of the ablation plume is taken into account, but its properties (i.e. density, velocity and angular distribution) are not calculated.

\section{$2 \quad$ Model \\ 2.1 Physical basics}

A laser beam (TEM 00 mode) is considered to be incident on a target surface and a Gaussian temporal profile is assumed for the laser pulse. These premises correspond to

Fax: +41-56/462-4154, E-mail: g.dumitru@fh-aargau.ch a large number of applications, where Q-switched lasers are used for material processing. The incident beam is reflected partially by the surface and the absorbed part is considered as the energy source of the occurring ablation processes. From the irradiated surface, the energy is transported into material through heat diffusion; the enthalpy form of this equation was considered, due to the fact that phase changes are induced by the absorbed laser energy (see, for instance, Sect. 2.2.4 in [13]):

$\nabla(k(T) \nabla T(r, z, t))+(1-R) I(r, t) A(r, z)=\frac{\mathrm{d} H(r, z, t)}{\mathrm{d} t}$,

where $T(r, z, t)$ and $H(r, z, t)$ are the temperature and enthalpy (pro volume) distributions, $I(r, t)$ is the incident laser intensity, $A(r, z)$ is the target absorptivity, $R$ represents the surface reflectivity and $k(T)$ is the thermal conductivity.

The system is first at room temperature, $T_{0}$. The volume $\Omega$, where the solution is sought, is taken large enough to simplify the boundary conditions, whereas no radial heat flow occurs through the boundary $r=0$ (i.e. the axis of symmetry):

$T\left(r_{\max }, z, t\right)=T\left(r, z_{\max }, t\right)=T_{0}$,

$\left.\frac{\partial T(r, z, t)}{\partial r}\right|_{r=0}=0$.

Due to intrinsic metal properties, the laser energy is completely absorbed within a thin layer $(\sim 100 \mathrm{~nm})$ below the boundary $\Sigma$, on which the laser beam impinges; this boundary is initially positioned in the plane $z=0$. Hence the enthalpy of this thin layer increases with the incident laser energy:

$\left.\frac{\partial H(r, z, t)}{\partial t}\right|_{\Sigma}=\left.\frac{\left(1-R_{\Sigma}\right) \partial I(r, t)}{\partial z}\right|_{\Sigma}$

and no absorption occurs in the volume $\Omega$; therefore (1) becomes

$k \Delta(T(r, z, t))=\frac{\mathrm{d} H(r, z, t)}{\mathrm{d} t}$.

The incident beam is shielded by the ablation plume, whose expansion is assumed spherical in a first approximation. The plume radius $R_{\mathrm{pl}}$ is related to the ablated volume, $V_{\mathrm{abl}}$ :

$R_{\mathrm{pl}}(t) \propto \sqrt[3]{\frac{\varrho_{\mathrm{s}}}{\varrho_{\mathrm{v}}} V_{\mathrm{abl}}(t)}$, 
where $\varrho_{\mathrm{s}}$ and $\varrho_{\mathrm{v}}$ are the mass densities of the solid and vapour phases. The global plume absorption effects are described by means of a simulated coefficient $\alpha_{\mathrm{pl}}$, which has to be estimated:

$$
I(t)=I_{0}(t) \exp \left(-\alpha_{\mathrm{pl}} R_{\mathrm{pl}}(t)\right) .
$$

\section{2}

\section{Computer implementation}

Numerical calculations are based on introducing finite differences in the heat diffusion (5) and in the boundary conditions (2)-(4). The Runge-Kutta-Gill algorithm was numerically implemented in FORTRAN routines to integrate the finite-difference equations.

The volume $\Omega$ was represented through a mesh of $80 \times 60$ cells (depth and radial coordinates) and the finite-difference equations were assigned to mesh nodes. The volume of $(R, z)$, the surface layer of a cylinder, was

$V_{\text {cell }}=2 \pi R \Delta R \Delta z$

where $\Delta R$ and $\Delta z$ are radial and depth mesh periods. $\Delta R$ and $\Delta z$ were chosen as $0.25 \mu \mathrm{m}$ and $\Delta t$ steps were set at $1 / 500$ from the considered pulse durations. Convergence tests were performed for given $\Delta R$ and $\Delta z$ by increasing $\Delta t$, and no convergence problems were revealed.

Temperatures and enthalpies of all mesh cells are calculated iteratively and the enthalpy of a given cell after $N$ iteration steps gives its phase. Initially all cells are in solid phase. When a cell enthalpy exceeds the latent melting heat (point A, Fig. 1) the cell is considered as a solid-liquid mixture. In point $\mathrm{B}$ the melting process is complete; the entire cell is considered as liquid and its temperature increases. When the latent vaporisation heat is reached (point C), the cell is a mixture of liquid and vapour. If cell vaporisation is complete (point $\mathrm{D}$ ), the laser beam can propagate through the cell.

The displacement of the absorption interface $\Sigma$ is more complicated, because a significant part of the material is removed as melt. Therefore, the enthalpy associated with point $\mathrm{D}$ is larger than the value actually needed to remove the cell material. A critical enthalpy $H_{\mathrm{cr}}$ (point $\mathrm{D}^{\prime}$ ) was introduced as a criterion for a displacement of $\Sigma$ : the cell material remains liquid up to $T_{\text {cr }}$ (Fig. 1) and after that a complete vaporisation is assumed.

The ablated volume $V_{\text {abl }}$ is calculated after each iteration step as the sum of all cell volumes from which material was

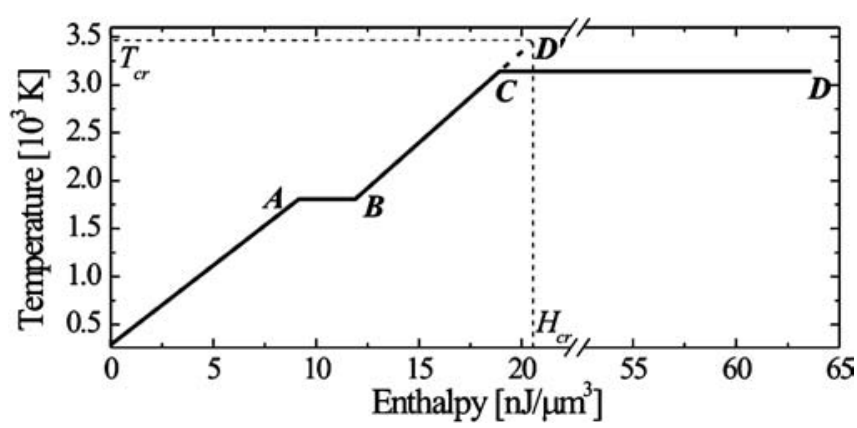

FIGURE 1 Temperature vs. enthalpy (pro volume) for steel removed. Using $V_{\mathrm{abl}}$ and (5) and (6), the shielding effects for the next iteration step are calculated.

\section{3}

\section{Parameter estimation}

Thermal and optical material constants were taken from the literature $[14,15]$ but the model-specific parameters $\left(H_{\mathrm{cr}}\right.$ and $\left.\alpha_{\mathrm{pl}}\right)$ had to be estimated. The estimation procedure was performed by comparing experimental results obtained with pulse energies of $50 \mu \mathrm{J}$ and $20 \mu \mathrm{J}$ (spot diameter of $25 \mu \mathrm{m}$, i.e. maximal fluences of $\sim 5 \mathrm{~J} / \mathrm{cm}^{2}$ and $\sim 32 \mathrm{~J} / \mathrm{cm}^{2}$ ) with model results obtained for the same laser parameters.

Guess values were firstly chosen in a logarithmic scale for $\alpha_{\mathrm{pl}}\left(10,31.6,100, \ldots \mathrm{cm}^{-1}\right)$ and with a $100-\mathrm{K}$ spacing $(3200$, $3300, \ldots$ K) for $T_{\mathrm{cr}}$. In a second phase, denser test values were chosen in the vicinities of the roughly estimated $\alpha_{\mathrm{pl}}$ and $T_{\mathrm{cr}}$; those yielding the smallest differences from experimental outcomes $\left(\alpha_{\mathrm{pl}}=250 \mathrm{~cm}^{-1}, T_{\mathrm{cr}}=3450 \mathrm{~K}\right)$ were used in the further simulations.

\section{4}

\section{Model verification}

Experimental investigations and simulations were performed for a different parameter set: pulse energy of $50 \mu \mathrm{J}$, spot diameter of $10 \mu \mathrm{m}$ and pulse duration of $100 \mathrm{~ns}$; this corresponds to a fluence of $\sim 127 \mathrm{~J} / \mathrm{cm}^{2}$ and to an intensity of $\sim 1.3 \times 10^{9} \mathrm{~W} / \mathrm{cm}^{2}$ (maximal values, Gaussian beam). Micropores were produced in a steel target (AISI M3) using a Nd : YAG laser, at the wavelength of $1.064 \mu \mathrm{m}$ (reflectivity of $60 \%-65 \%[14,15])$. The target was afterwards cut in cross section. The new surfaces were etched (Fig. 2) and it was possible to determine the extent of the zone where material melting occurred. The observed ablation depth $(\sim 10 \mu \mathrm{m} /$ pulse $)$ and pore form are related [16] to the high peak fluence; the material expulsion through the vapour pressure occurs in a quasi-normal direction to the surface and it is more efficient.

Figure 2 shows a cross-sectional surface after etching, where two micropores induced in alloyed steel can be seen. In this figure the white bands surrounding the craters can be identified as thermally affected zones. Results of model calculations $(12 \mu \mathrm{m} \times 8 \mu \mathrm{m}$ rectangles, in which pore profiles and melt depths are shown) are superimposed on the microscope images. Between the calculated and the actual crater forms and between the estimated melt depth and the extension of the white zone, a good agreement can be noticed.

\section{$3 \quad$ Simulations}

Different FWHM durations were considered for the incident laser pulses: 500, 100, 20, 5 and $1 \mathrm{~ns}$. The processes were simulated over intervals that were four times larger than the corresponding pulse durations; each interval was centred on the moment of maximal laser intensity. Calculations were performed for incident pulse energies of $50 \mu \mathrm{J}$ and for spot diameters of $10 \mu \mathrm{m}$.

Typical simulation outcomes are shown in Fig. 3. In the left-hand panel of this figure, the temporal evolution of the pore profile is depicted as contour lines of constant depths, whereas the end pore profile and the estimated extension of the melted zone are given in the right-hand panel. 


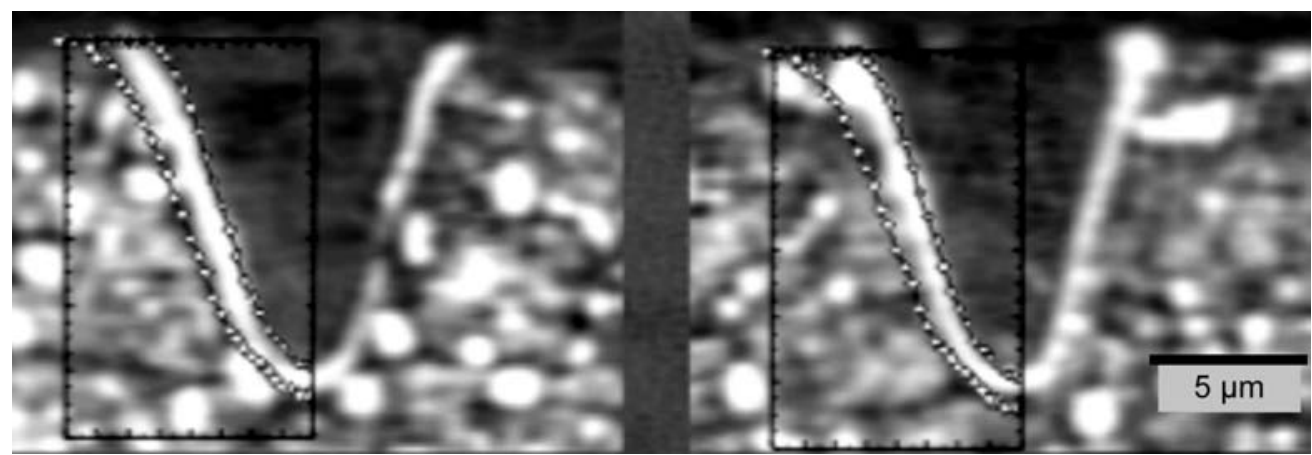

FIGURE 2 Results of numerical simulations (pore boundary and melt limit) superimposed on images of laser-induced microcraters in AISI M3 steel $(1064 \mathrm{~nm}, 100 \mathrm{~ns}$, $\sim 127 \mathrm{~J} / \mathrm{cm}^{2}$ )
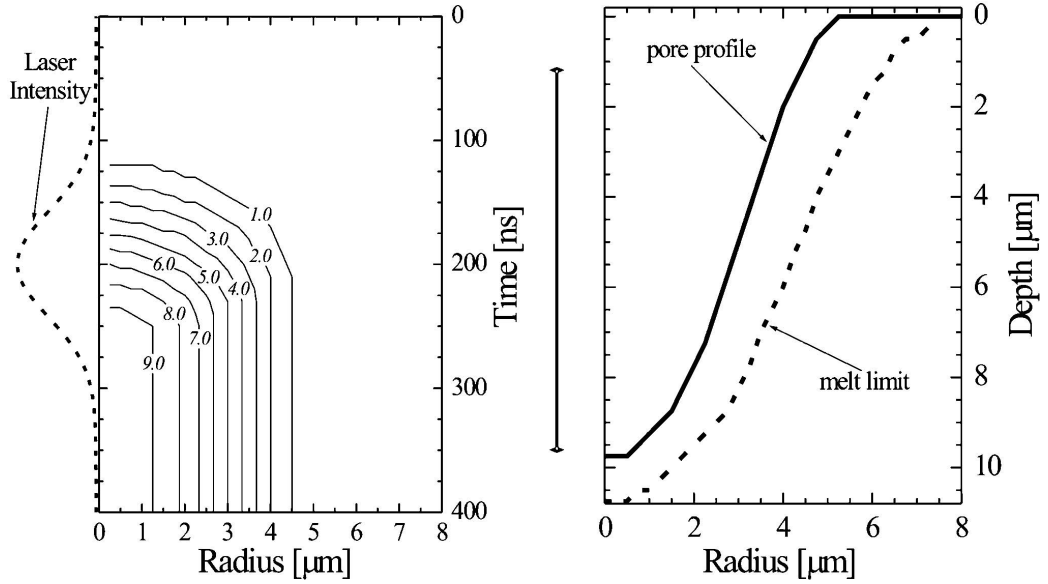

FIGURE 3 Typical simulation outcomes: temporal depth evolution (left, contour lines are depth values in $\mu \mathrm{m}$ ); calculated pore boundary and melt limit (right)
4

4.1

Results

Temporal evolution of the ablated volume

The curves characterising the temporal evolution of the ablated volume are shown in Fig. 4. Similar process developments can be observed for all pulse durations: material removal begins after an accumulation time, then the ablated volume increases with the deposited energy and finally the ablated volume does not increase any more, even though the laser beam is still irradiating the target.

The curves corresponding to 20-, 5- and 1-ns pulses have almost identical evolutions: saturation after 2.90 FHWM units and maximal ablated volumes. For 100-ns pulses, the saturation occurs earlier (2.53 FHWM units) and the final pore volume represents $90.1 \%$ from maximum. In the case of 500-ns pulses, material removal is temporally retarded, saturation occurs after 2.31 FHWM units and the final ablated volume is only $47.4 \%$ of the maximal value. Hence, the amplitude of en-

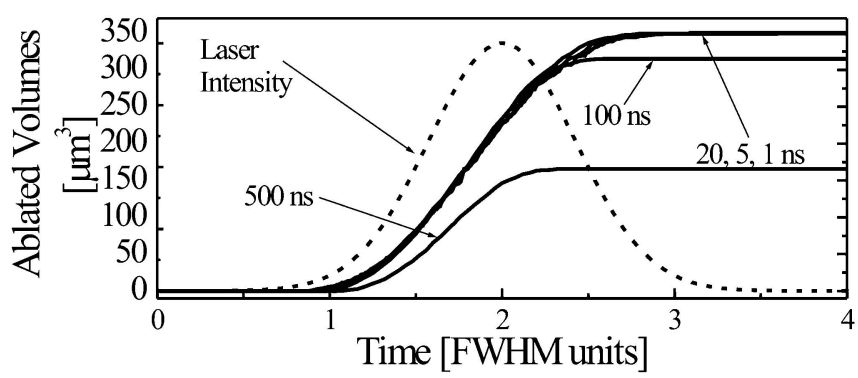

FIGURE 4 Calculated ablated volumes for different pulse durations; pulse energy: $50 \mu \mathrm{J}$, spot diameter: $10 \mu \mathrm{m}$ (i.e. $\sim 125 \mathrm{~J} / \mathrm{cm}^{2}$ ) ergy losses through heat diffusion is significant in the case of 500-ns pulses.

Using the above-determined $V_{\mathrm{abl}}$ and (6) and (7), the fraction of laser energy absorbed in the ablation plume at different times can be calculated. For $\leq 100 \mathrm{~ns}$ pulses, fractions of $50 \%-55 \%$ were determined at maximal laser intensities, whereas absorption values up to $65 \%-70 \%$ were found with increasing $V_{\mathrm{abl}}$. In the 500-ns case, a value of $\sim 40 \%$ was calculated at maximal intensity and (see Fig. 4) no significant further increase occurred.

\subsection{Extension of the melt zone}

Calculated pore profiles and melt-zone extensions are shown in Fig. 5. The extension of the melt zone diminishes with decreasing pulse duration $(500,100$ and $20 \mathrm{~ns})$ and further decreases ( 5 and $1 \mathrm{~ns}$ ) in pulse duration yielded no more changes. The pore profiles calculated for 20,5 and $1 \mathrm{~ns}$ are almost identical; a thin melt zone still appears at $20 \mathrm{~ns}$, whereas for 5 and $1 \mathrm{~ns}$ vanishing melt-zone extensions were found.

This indicates that optimal pulse durations for ns laser microprocessing are situated below $20 \mathrm{~ns}$, roughly around $10 \mathrm{~ns}$. For these pulse durations plasma shielding and heat-diffusion losses were found minimal and only thin $(<0.25 \mu \mathrm{m})$ melt zones were calculated.

5

\section{Conclusions}

A numerical model based on an enthalpy scheme was developed and the evolution of several process parameters related to pulsed laser drilling was calculated during and after the working pulse. 

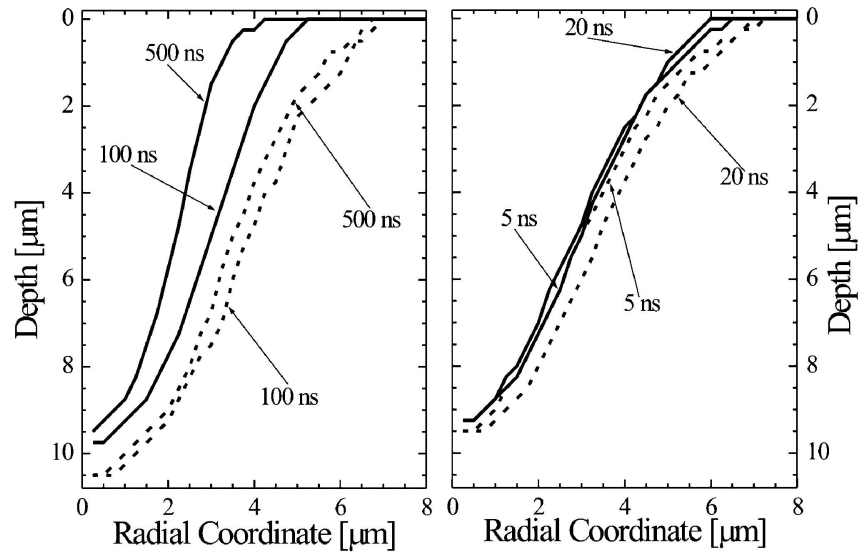

FIGURE 5 Calculated end pore profiles and melt-zone extensions for different pulse durations; pulse energy: $50 \mu \mathrm{J}$, spot diameter: $10 \mu \mathrm{m}$ (i.e. $\sim 125 \mathrm{~J} / \mathrm{cm}^{2}$ )

The stoppage of the ablation process was evidenced before the end of the laser pulse. This could be correlated with the shielding effect of the ablation plume, which is continuously growing and whose absorption is increasing.

Calculations performed with different pulse durations indicated the amplitude of the thermal effects. For the smallest considered pulse durations (5 and $1 \mathrm{~ns}$ ), the thermal effects are limited by the intrinsic characteristics of ns laser ablation; maximal ablated volumes were found. In the case of the 20-ns pulse, maximal pore volumes were also found, but the extension of the melt zone is not negligible any more. The energy lost by diffusion during the pulse begins to play a role for 100-ns and becomes significant for 500-ns pulse durations. The maximal extension of the melt zone was found for 500-ns pulses.

ACKNOWLEDGEMENTS G. Dumitru acknowledges the support received from the University of Applied Sciences Aargau during the final phase of manuscript preparation.

\section{REFERENCES}

1 A. Bächli, A. Blatter: Refract. Met. Hard Mater. 11, 113 (1992)

2 S. Gasser, V. Romano, R. Weber, H.P. Weber, A. Blatter: Proc. ISATA 1994, 367 (1995)

3 S. Fähler, H.-U. Krebs: Appl. Surf. Sci. 96-98, 61 (1996)

4 J. Lunney, R. Jordan: Appl. Surf. Sci. 127-129, 941 (1998)

5 R.F. Wood, K.R. Chen, J.N. Leboeuf, A.A. Puretzky, D.B. Geohegan: Phys. Rev. Lett. 79, 1571 (1997)

6 A. Mele, A. Giardini Guidoni, R. Kelly, C. Flamini, S. Orlando: Appl. Surf. Sci. 109, 584 (1997)

7 N. Arnold, J. Gruber, J. Heitz: Appl. Phys. A 69, S87 (1999)

8 R. Singh: J. Electron. Mater. 25, 125 (1996)

9 J.N. Leboeuf, K.R. Chen, J.M. Donato, D.B. Geohegan, C.L. Liu, A.A. Puretzky, R.F. Wood: Phys. Plasmas 3, 2203 (1996)

10 A. Misra, A. Mitra, R.K. Thareja: Appl. Phys. Lett. 74, 929 (1999)

11 X. Mao, W.T. Chan, M. Caetano, M.A. Shannon, R.E. Russo: Appl. Surf. Sci. 96-98, 126 (1996)

12 B. Toftmann, J. Schou, T.N. Hansen, J.G. Lunney: Phys. Rev. Lett. 84, 3998 (2000)

13 D. Bäuerle: Laser Processing and Chemistry (Springer, Berlin 2000)

14 M. von Allmen, A. Blatter: Laser-Beam Interactions with Materials (Springer, Berlin 1995)

15 J.F. Ready, D.F. Farson (Eds.): Handbook of Laser Materials Processing (LIA, Orlando 2001)

16 G. Dumitru, V. Romano, H.P. Weber, H. Haefke, Y. Gerbig: Appl. Phys. A 70, $485(2000)$ 